\title{
Digitalization of the world economy - a factor in the development of society
}

\author{
Peter Grabovyy ${ }^{1, *}$ \\ ${ }^{1}$ Moscow State University of Civil Engineering, 129337, 26, Yaroslavskoye Shosse, Moscow, Russia
}

\begin{abstract}
Another restructuring is underway in Russia, this time digital. Almost all sectors of the economy began to transform in order to keep up with progress and increase their efficiency. The development of the digital economy in the country will improve the quality of life of citizens and increase the global competitiveness of domestic industries. The state began its activities in this direction with the formation of the Digital Economy program, which was developed in 2017 by the joint efforts of the state, business and the expert community. The article presents the main indicators of the development of the digital economy in Russia and its position in international ratings. The indicators characterizing research and development in the field of ICT; personnel of the digital economy; ICT, content and media sectors; telecommunications development.
\end{abstract}

\section{Introduction}

At the beginning of the XXI century. humanity has been swept by a wave of serious global changes. This stage is distinguished by the breakthrough development of digital technologies, a revolution in the information space, and acceleration of the globalization of the economy. Information has acquired the status of a key resource in public and business processes [1-3]. The penetration of digital technology into life is one of the characteristic features of the future world. This is due to progress in the fields of microelectronics and telecommunications, IT-technologies. The transition to the rails of digitalization is today one of the key priorities in the development of the global economy.

By 2024, the state intends to carry out a comprehensive digital transformation of the Russian economy and social sphere. To do this, it is necessary to develop legislation on digital technologies, modernize the digital infrastructure, introduce digital practices in all key areas of the economy and government, and organize training for the transition period. The national project "Digital Economy of the Russian Federation" is called upon to provide an integrated approach to solving these issues.

The main goal of the project, the implementation of which is designed for 6 years, is the introduction of digital technologies in various structures of society: economy, industry, urban economy, social sphere and public administration. The program is designed to increase the efficiency of various types of industries due to the transition to digital

*Corresponding author: meshcheryakovats@mgsu.ru 
technologies, which will allow in the future to carry out a large amount of work with less time and resource costs,

\section{Materials and Methods}

The study analyzed data from the International Telecommunication Union (ITU), Department of Economic and Social Affairs (UN DESA), which formed the international rating systems for assessing the digitalization level of the pestilential market.

The program documents on the digital economy of the Russian Federation were taken on the platform of the ANO "Digital Economy" (a platform for dialogue between business and the state), which performs the following functions: - forms and coordinates the activities of the working group, defines and coordinates the activities of the center of competence; - assesses the effectiveness of the implementation of the Program; - prepares proposals on the main areas of development of the digital economy.

The following scientific methods were used for research: analysis - decomposition of a single system into its constituent parts and their study separately, which allows to evaluate individual elements of the Digital Economy program; - synthesis - combining all the results of the analysis into a single system, which allows us to provide a general idea of the current level of digitalization of the Russian economy; - an analogy is a conclusion about the similarity of two objects in a particular attribute based on their similarity in other characteristics, which is used to compare the digitalization level of different countries.

\section{Results}

The creation of institutional and infrastructural conditions, the elimination of existing obstacles and restrictions for the creation and development of high-tech businesses is carried out in 6 areas:

1. Personnel for the digital economy. Improving the education system, which should provide the digital economy with competent personnel. The transformation of the labor market, which should be based on the requirements of the digital economy. Creating a motivation system for the development of the necessary competencies and the participation of personnel in the development of the digital economy of Russia

2. Information infrastructure. The development of communication networks, the development of a system of Russian data centers, the introduction of digital platforms for working with data to meet the needs of citizens, business and government.

3. Information security. Achieving the state of protection of the individual, society and the state from internal and external information threats, which ensures the realization of constitutional rights and freedoms of man and citizen, worthy of the quality and standard of living of citizens, sovereignty and sustainable socio-economic development of the Russian Federation.

4. Digital technology. Creation of a support system for search and applied research in the field of the digital economy (research infrastructure of digital platforms), ensuring technological independence in each of the areas of end-to-end digital technologies that are globally competitive and national security.

5. Normative regulation. Formation of a new regulatory environment that provides a favorable legal regime for the emergence and development of modern technologies, as well as for the implementation of economic activities related to their use.

6. Digital government. Introduction of digital technologies and platform solutions in the areas of public administration and the provision of public services, including in the interests 
of the population and small and medium-sized enterprises, including individual entrepreneurs

The tasks of the "Personnel and Education" direction are to ensure citizens are prepared for the conditions of the digital economy and train professionals who can cope with the challenges of digital transformation. A promising way to solve these problems is the development of a culture of self-education, taking into account competencies that are relevant for the digital economy. Based on the results of the implementation of the plan, the number of university graduates in IT specialties should grow from 46 thousand people in 2017 to 120 thousand in 2024. The profile "Competence Center" of the Agency for Strategic Initiatives for the Promotion of New Projects is defined by the Profile Center of Competencies.

An important task of the direction "Information Infrastructure" is to provide citizens and devices of the Internet of things with Internet access in the country. In terms of measures for the next three years - the Internet connection of all schools and medical institutions, the development of new technologies for wireless Internet connection. We are talking about 5G technology, about the program and the development of action plans. The development of the digital economy in the country will improve the quality of life of citizens and increase the global competitiveness of domestic industries. The state began its activities in this direction with the formation of the Digital Economy program, which was developed in 2017 by the joint efforts of the state, business and the expert community. The role of ANO "Digital Economy" was to organize a dialogue with business and expert communities on the development of draft action plans for the Program for 2018-2020. and subsequent monitoring of their implementation. The plan includes the development of data centers. By 2024 , the proportion of households with broadband Internet access should reach $97 \%$, the speed of the Internet with stable coverage throughout the country will be at least $100 \mathrm{Mbit} /$ $\mathrm{s}$, and in cities with over 1 million people a stable coverage of the $5 \mathrm{G}+$ communication standard will be achieved.

The main task of the "Information Security" direction is to ensure the literacy of the population in the new digital world and the support of Russian manufacturers, so that a significant part of the solutions will be implemented on domestic platforms. As a result of the work of the direction, by 2024 it is planned to increase the proportion of citizens who have increased literacy in the field of information security by at least $50 \%$, the share of Internet users using security equipment - up to $97 \%$.

Heads of R\&D departments of large companies, leading universities, and representatives of the Russian Academy of Sciences were involved in the work of the "Formation of Research Competencies and Scientific and Technological Departments" direction. As part of the Digital Economy, a stake has been placed on the so-called end-toend technologies, which in the coming years will affect the transformation of almost all sectors of the economy: big data, artificial intelligence, quantum technologies, etc.

The work of the direction "Normative regulation" can be called the driving force of legal reform, which has already begun. With the participation of experts, a new regulatory environment is being formed that provides a favorable legal regime for the emergence and development of modern technologies. This is the removal of legal barriers to the development of the digital economy, the development of electronic civil circulation and much more.

A necessary attribute of economic transformation is the big data strategy, which is being developed by the Association of Big Data Market Participants, combining: MegaFon, Rostelecom, Yandex, Mail.ru Group, Sberbank, Tinkoff Bank, Gazprombank, etc. Also in development involved by the Boston Consulting Group. At present, the Big Data Strategy for 5 years (until 2024) has been approved. 
The main goal of the strategy is to create "rules of the game": the formed model must meet the needs of all three parties of legal relations - citizens, business and the state. Without such rules, it will be impossible to implement an artificial intelligence development strategy.

Currently, the domestic big data market is estimated at 45 billion rubles, and its average annual growth over the past few years is $12 \%$. According to the Association, in a few years it could well be about 100 billion rubles. This will require overcoming a number of barriers - infrastructural, human, legal, related to data availability and scaling of projects, etc. This is exactly what the big data market strategy is aimed at. Within the framework of the strategy, 18 projects and three programs were combined - the Data Accessibility Improvement Program, the R\&D Program and the Market Scaling Program. Their implementation will take about two years.

Russia is not a unique country that has created a new structure for the development of the big data market. Similar initiatives are typical for other countries: the problem of creating a data market and its regulation is relevant for everyone.

To analyze the global digitalization market, we use:

- Index of development of information and communication technologies (ICT Development Index) [4,6];

- E-Government Development Index [5,6];

- The Global Cybersecurity Index (Global Cybersecurity Index) [4,6].

ICT Development Index is a combined indicator characterizing the achievements of the countries of the world in terms of the development of information and communication technologies (ICT). It is calculated according to the methodology of the International Telecommunication Union (International Telecommunication Union), a specialized UN unit that defines world standards in the field of ICT. The index was developed in 2007 based on 11 indicators used by the International Telecommunication Union in its assessments of ICT development. The index brings these indicators into a single criterion, which is designed to compare the achievements of the countries of the world in the development of ICT and can be used as a tool for conducting comparative analysis at the global, regional and national levels. These indicators relate to access to ICT, the use of ICT, as well as skills, that is, practical knowledge of these technologies by the population of the countries covered by the study. Here are the results of the rating of countries according to the ICT Development Index (table 1).

Table 1. Information and communications technology development index in the countries.

\begin{tabular}{|c|c|c|}
\hline Rating & Country & Index \\
\hline 1 & Iceland & 8.98 \\
\hline 2 & South Korea & 8.85 \\
\hline 3 & Switzerland & 8.74 \\
\hline 4 & Denmark & 8.71 \\
\hline 5 & United Kingdom & 8.65 \\
\hline 6 & Hong Kong & 8.61 \\
\hline 7 & Netherlands & 8.49 \\
\hline 8 & Norway & 8.47 \\
\hline 9 & Luxembourg & 8.47 \\
\hline 10 & Japan & 8.43 \\
\hline
\end{tabular}

Russia occupies 45th position in this ranking, and is located after Portugal. Russia has a fairly low position in other indices that characterize the level of digitalization of the national economy (Figure 1). 


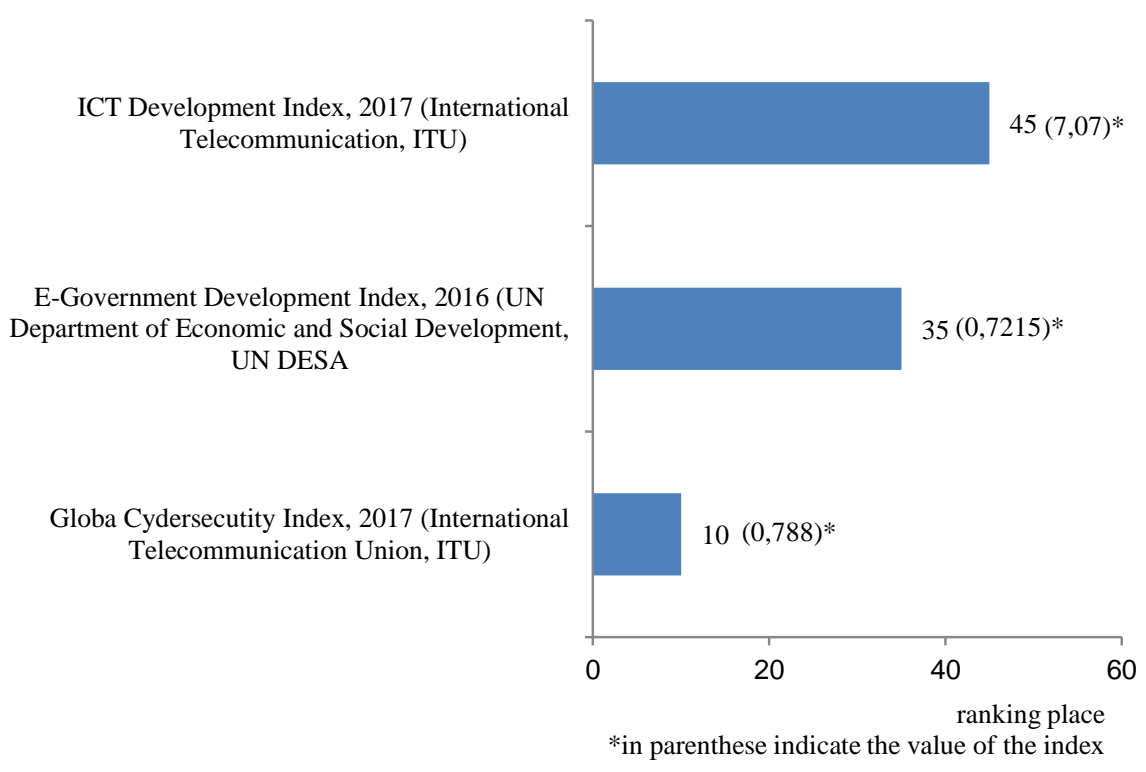

Fig. 1. Russia's place in international ratings for the development of the digital economy.

The E-Government Development Index is presented according to UN DESA (Figure 2).

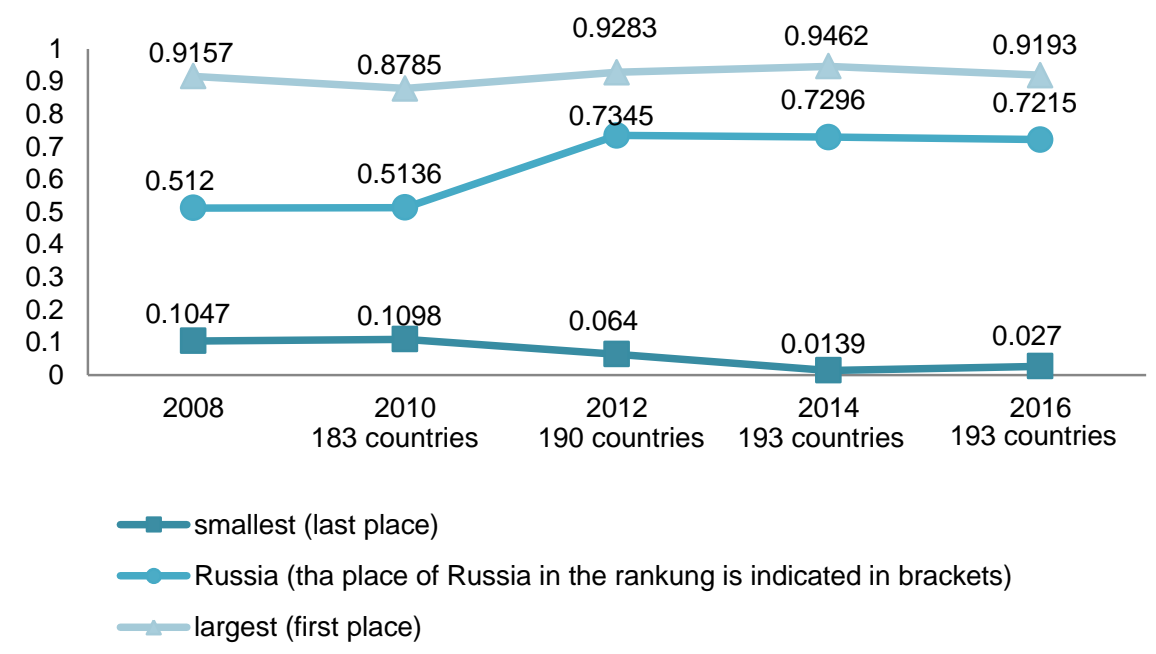

Fig. 2. E-Government Development Index.

The Global Cybersecurity Index includes the following subindexes:

- Legal aspects of cybersecurity (Legal);

- Technical aspects of cybersecurity (Technical);

- Organizational aspects of cybersecurity (Organizational);

- Country skills in building a cybersecurity system (Capacity Building);

- International cooperation in the field of cybersecurity (Cooperation).

The latest statistics determining the global cybersecurity index by country are presented for 2017. According to these data, Russia is in 10th place in the ranking (Figure 3). 


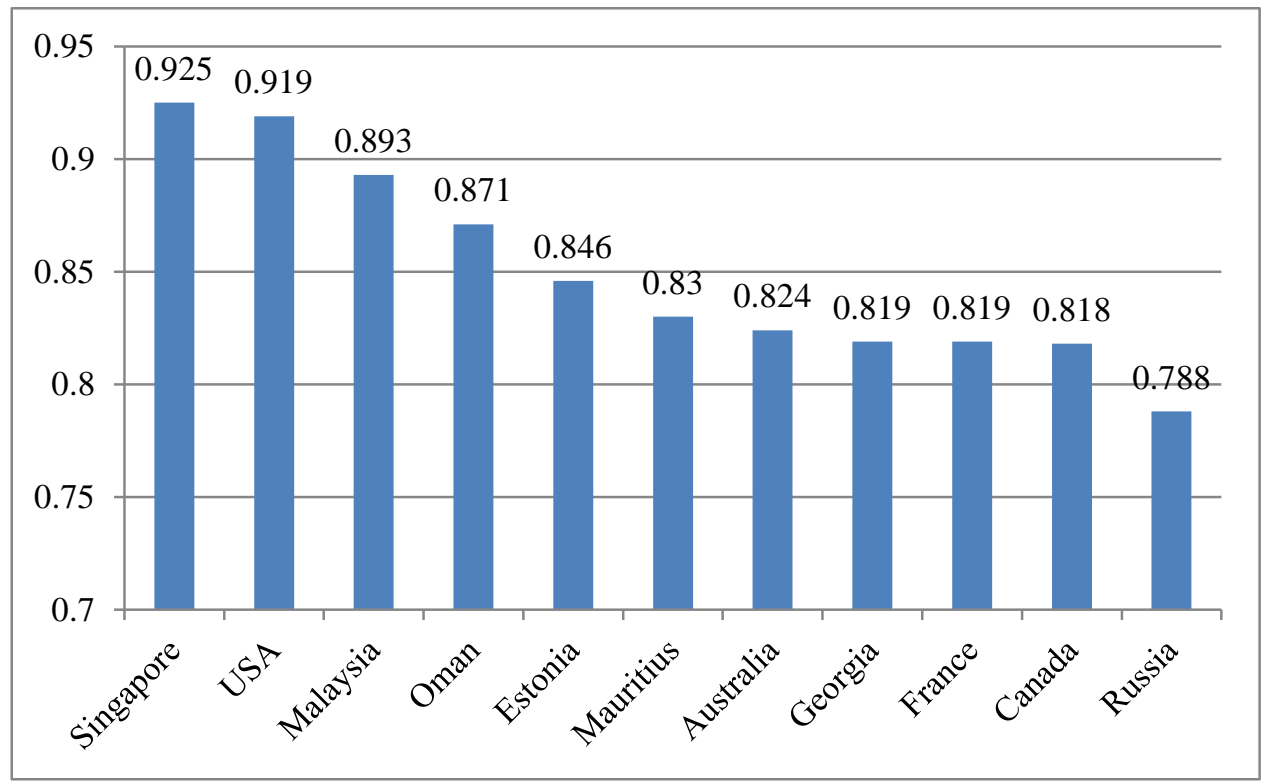

Fig. 3. Global cyber security index.

\section{Discussion}

Application of the Internet of things technology in industry provides monitoring, control and analysis of the operation of industrial equipment, materials and processes, as well as electricity consumption with a high degree of detail and in real time. In the field of housing and communal services, IoT (Internet of Things technology) is used to create "smart homes" that are provided with an automated system of accounting for consumed resources and protected by an intelligent security system. Solutions based on the Internet of things in retail will allow you to use data array analysis to quickly analyze the market, trends and preferences of customers.

In an urban environment, the use of IoT can ensure the development of a "Safe City", which will be equipped with an intelligent automated security and control system, centralized video surveillance to identify threats to public safety, and a monitoring system for basic natural and technological threats. In the field of healthcare, the Internet of things allows you to create intelligent remote health monitoring systems that collect and analyze information about the patient's health, increasing the accuracy of diagnosis and treatment. IoT technologies in agriculture will make accurate the consumption of seeds, agricultural chemistry, fertilizers and water, increase the efficiency of land use, and automate agricultural work.

\section{Conclusion}

In fact, the digital economy is a living organism that is constantly evolving, so the Program has a very flexible mechanism for updating plans, which allows you to quickly respond to all changes in the outside world. At the moment, a foundation has been formed within the framework of the Program, while market and industry directions and regional activities have not been covered. Nowadays the directions "Digital Healthcare" and "Digital Transport and Logistics" are being worked out, in which high-tech companies, enterprises 
of various industries, experts and government representatives participate. The work is carried out in the logic of data economics, with the analysis of proposals for industry platforms and services.

The development of the big data market in Russia even in the most pessimistic scenario is capable of bringing in at least $0.3 \%$ of GDP or 1.3 trillion rubles in the next five years. This is $15 \%$ of revenue from current oil exports. The numbers were not taken "from the ceiling": to assess the market potential, Boston Consulting Group analysts were involved, with the help of which about 500 scenarios of using big data in various industries were analyzed and based on this a special calculator was developed.

Basic and optimistic scenarios imply a much more noticeable effect - from $1.2 \%$ to $1.8 \%$ of GDP. To implement them, it will be necessary to solve several fundamentally new problems, including enriching user data (exchanging them between business and the state), exchanging industry data, wide access to data lakes, creating investment platforms for startups and removing barriers to exporting big data.

\section{Reference}

1. T. Meshcheryakova, MATEC Web of Conf., 170, 01048 (2018) doi: 0.1051/matecconf/201817001048

2. T. Meshcheryakova, IOP Conf. Ser.: Earth and Envir. Scie., 90, 012118 (2017) doi :10.1088/1755-1315/90/1/012118

3. Y.I. Dubova, Advances in intelligent systems and computing, 726, pp. 386-392 (2019) doi: 10.1007/978-3-319-90835-9_45

4. International Telecommunication Union, Measuring the Information Society Report was launched during the World Telecommunication/ICT Indicators Symposium (WTIS) (Hammamet, Tunisia, 2017)

5. Department of Economic and Social Affairs, UN E-Government Survey 2018 (UNITED NATIONS, New York, 2018)

6. Digital Economy Indicators: 2018. Statistical Digest (National Research University Higher School of Economics, Moscow, 2018) 